\title{
A characterization of generalized exponential polynomials in terms of decomposable functions
}

\author{
Miklós Laczkovich (Budapest, Hungary)
}

December 18, 2018

\begin{abstract}
Let $G$ be a topological commutative semigroup with unit. We prove that a continuous function $f: G \rightarrow \mathbb{C}$ is a generalized exponential polynomial if and only if there is an $n \geq 2$ such that $f\left(x_{1}+\ldots+x_{n}\right)$ is decomposable; that is, if $f\left(x_{1}+\ldots+x_{n}\right)=\sum_{i=1}^{k} u_{i} \cdot v_{i}$, where the function $u_{i}$ only depends on the variables belonging to a set $\emptyset \neq E_{i} \subsetneq\left\{x_{1}, \ldots, x_{n}\right\}$, and $v_{i}$ only depends on the variables belonging to $\left\{x_{1}, \ldots, x_{n}\right\} \backslash E_{i}(i=1, \ldots, k)$.
\end{abstract}

\section{Introduction and main results}

Let $X$ be a nonempty set. A function of $n$ variables $F: X^{n} \rightarrow \mathbb{C}$ is called decomposable of order $k$ if there are functions $u_{i}, v_{i}: X^{n} \rightarrow \mathbb{C}(i=1, \ldots, k)$ such that $F=\sum_{i=1}^{k} u_{i} \cdot v_{i}$ and, for every $i=1, \ldots, k, u_{i}$ only depends on the variables belonging to a set $\emptyset \neq E_{i} \subsetneq\left\{x_{1}, \ldots, x_{n}\right\}$, and $v_{i}$ only depends on the variables belonging to $\left\{x_{1}, \ldots, x_{n}\right\} \backslash E_{i}$. For example, if $G$ is a semigroup and $f: G \rightarrow \mathbb{C}$ satisfies a Levi-Civita equation $f(x y)=\sum_{i=1}^{k} a_{i}(x) \cdot b_{i}(y)$ $(x, y \in G)$, then $f(x y)$ is decomposable of order $k$.

The following remarkable result was proved by Ekaterina Shulman in [5, Theorem 6]. Let $G$ be a topological semigroup with unit. If a continuous 
function $f: G \rightarrow \mathbb{C}$ is such that $f\left(x_{1} \cdots x_{n}\right)$ is decomposable for some $n>1$, then $f$ is an almost matrix function.

A function is a matrix function if $f(x y)$ satisfies a Levi-Civita equation. The function $f$ is an almost matrix function if, for every finite set $E \subset G$, there is a finite dimensional subspace of $C(G)$ containing $f$ and invariant under the subsemigroup generated by $E$. It was also shown by Shulman that if $G$ is the union of an increasing net of topologically p-generated subsemigroups, then, whenever $f \in C(G)$ is such that $f\left(x_{1} \cdots x_{n}\right)$ is decomposable for some $n>1$, then $f$ is a matrix function. In particular, if $f \in C\left(\mathbb{R}^{p}\right)$ is such that $f\left(x_{1}+\ldots+x_{n}\right)$ is decomposable for some $n>1$, then $f$ is an exponential polynomial ([5, Corollary 8]).

In this note our aim is to prove that in the commutative case, if $f\left(x_{1}+\right.$ $\left.\ldots+x_{n}\right)$ is decomposable, then $f$ is necessarily a generalized exponential polynomial. The relevant definitions are as follows.

Let $G$ be an Abelian topological semigroup with unit, written additively. Let $C(G)$ denote the set of complex valued continuous functions defined on $G$. The difference operator $\Delta_{h}$ is defined by $\Delta_{h} f(x)=f(x+h)-f(x)$ $(x \in G)$ for every $h \in G$ and $f: G \rightarrow \mathbb{C}$. A function $f \in C(G)$ is a generalized polynomial, if there is an $n \geq 0$ such that $\Delta_{h_{1}} \ldots \Delta_{h_{n+1}} f=0$ for every $h_{1}, \ldots, h_{n+1} \in G$. The smallest $n$ with this property is the degree of $f$, denoted by $\operatorname{deg} f$. The degree of the identically zero function is -1 . The set of generalized polynomials will be denoted by GP.

A function $m \in C(G)$ is an exponential, if $m \neq 0$ and $m(x+y)=$ $m(x) \cdot m(y)$ for every $x, y \in G$. The functions of the form $\sum_{i=1}^{s} p_{i} \cdot m_{i}$, where $p_{i} \in \mathrm{GP}$ and $m_{i}$ is an exponential for every $i=1, \ldots, s$ are called generalized exponential polynomials. The set of generalized exponential polynomials will be denoted by GEP.

In the rest of the paper we assume that $G$ is a commutative unital semigroup written additively. Our main result is the following.

Theorem 1. For every continuous function $f \in C(G)$ the following are equivalent.

(i) There is an $n \geq 2$ such that $f\left(x_{1}+\ldots+x_{n}\right)$ is decomposable. 
(ii) $f$ is a generalized exponential polynomial.

It is easy to see that if $f\left(x_{1}+\ldots+x_{n}\right)$ is decomposable, then so is $f\left(x_{1}+\ldots+x_{n^{\prime}}\right)$ for every $n^{\prime}>n$. Thus (i) is equivalent to the statement that $f\left(x_{1}+\ldots+x_{n}\right)$ is decomposable for every $n$ large enough.

Note that the class GEP is contained in the class of almost matrix functions, and the containment, in general, is strict (see [5, example 1, p. 18]). Therefore, Theorem 1 is more precise than [5, Theorem 6] (in the commutative case).

Let $f=\sum_{i=1}^{s} p_{i} \cdot m_{i}$, where $m_{1}, \ldots, m_{s}$ are distinct exponentials and $p_{1}, \ldots, p_{s}$ are nonzero generalized polynomials. Then the degree of $f$ is defined by $\operatorname{deg} f=\sum_{i=1}^{s}\left(1+\operatorname{deg} p_{i}\right)$ if $m_{i} \neq 1$ for every $i=1, \ldots, s$, and by $\operatorname{deg} f=$ $-1+\sum_{i=1}^{s}\left(1+\operatorname{deg} p_{i}\right)$ if $m_{i}=1$ for one of the $i$ 's. If $f=0$, then we put $\operatorname{deg} f=-1$.

It is well-known that every $f \in \mathrm{GEP}, f \neq 0$ has a unique representation of the form $\sum_{i=1}^{s} p_{i} \cdot m_{i}$, where $m_{1}, \ldots, m_{s}$ are distinct exponentials and $p_{1}, \ldots, p_{s}$ are nonzero generalized polynomials. (For Abelian groups this is proved in [6, Lemma 4.3, p. 41] and in [4, Lemma 6]. It is easy to check that the proof of [4, Lemma 6] works in Abelian semigroups as well.) Thus $\operatorname{deg} f$ is well-defined for every $f \in$ GEP. It is clear that $\operatorname{deg} f$ equals the usual degree of $f$ if $f \in \mathrm{GP}$.

The following result gives the direction (ii) $\Longrightarrow($ i) of Theorem 1 .

Theorem 2. Let $f=\sum_{i=1}^{s} p_{i} \cdot m_{i}$, where $p_{i} \in \mathrm{GP}, p_{i} \neq 0(i=1, \ldots, s)$, and $m_{1}, \ldots, m_{s}$ are distinct exponentials. Put $n_{0}=\max _{1 \leq i \leq s} \operatorname{deg} p_{i}$ and $k=$ $\operatorname{deg} f$.

(i) If $m_{i} \neq 1$ for every $i=1, \ldots, s$, then $f\left(x_{1}+\ldots+x_{n}\right)$ is decomposable of order $k$ for every $n>n_{0}$.

(ii) If $m_{i}=1$ for one of the indices $i=1, \ldots, s$, then $f\left(x_{1}+\ldots+x_{n}\right)$ is decomposable of order $k+1$ for every $n>n_{0}$.

Proof. It is enough to prove the theorem in the special case when $s=1$. 
The function of $i$ variables $A: G^{i} \rightarrow \mathbb{C}$ is called $i$-additive, if it is additive in each of its variables (the other variables being fixed). A function $f_{i}: G \rightarrow$ $\mathbb{C}$ is a monom of degree $i$, if there exists a symmetric and $i$-additive function $A_{i}$ such that $f_{i}(x)=A_{i}(x, \ldots, x)$ for every $x \in G$.

Let $p \in \mathrm{GP}$ be given, and let $\operatorname{deg} p=k$. Then $p=\sum_{i=0}^{k} f_{i}$, where $f_{i}$ is a monom of degree $i$ for every $i=1, \ldots, k$, and $f_{0}$ is constant. (See [3, Theorem 3].) Suppose $f_{i}(x)=A_{i}(x, \ldots, x)(x \in G)$, where $A_{i}$ is symmetric and $i$-additive for every $i=1, \ldots, k$. If $1 \leq i \leq k$, then

$$
\begin{gathered}
f_{i}\left(x_{1}+\ldots+x_{k+1}\right)=A_{i}\left(x_{1}+\ldots+x_{k+1}, \ldots, x_{1}+\ldots+x_{k+1}\right)= \\
=\sum_{j_{1}, \ldots, j_{i}=1}^{k+1} A_{i}\left(x_{j_{1}}, \ldots, x_{j_{i}}\right) .
\end{gathered}
$$

Since $i<k+1$, each term of the sum depends on the variables belonging to a proper subset of $\left\{x_{1}, \ldots, x_{k+1}\right\}$. Grouping the terms appropriately we find that

$$
f_{i}\left(x_{1}+\ldots+x_{k+1}\right)=\sum_{j=1}^{k+1} u_{i, j} \quad(i=1, \ldots, k),
$$

where $u_{i, j}$ does not depend on $x_{j}(j=1, \ldots, k+1)$. Putting $u_{j}=\sum_{i=1}^{k} u_{i, j}+$ $\left(f_{0} /(k+1)\right)$, we obtain

$$
p\left(x_{1}+\ldots+x_{k+1}\right)=f_{0}+\sum_{i=1}^{k} \sum_{j=1}^{k+1} u_{i, j}=\sum_{j=1}^{k+1} u_{j} \cdot 1
$$

where $u_{j}$ does not depend on $x_{j}(j=1, \ldots, k+1)$. Therefore, if $p \in$ GP and $\operatorname{deg} f=k$, then $p\left(x_{1}+\ldots+x_{k+1}\right)$ is decomposable of order $k+1$, and then so is $p\left(x_{1}+\ldots+x_{n}\right)$ for every $n>k$.

Let $f=p \cdot m$, where $p \in \mathrm{GP}, \operatorname{deg} p=k \geq 0$ and $m$ is an exponential. If $m=1$, then $\operatorname{deg} f=k$, and we obtain that (ii) holds in this special case.

Suppose $m \neq 1$. Multiplying (11) by $m\left(x_{1}+\ldots+x_{k+1}\right)=m\left(x_{1}\right) \cdots m\left(x_{k+1}\right)$, we find that $f\left(x_{1}+\ldots+x_{k+1}\right)$ is decomposable of order $k+1$, and then the same is true for $f\left(x_{1}+\ldots+x_{n}\right)$ for every $n>k$. By $m \neq 1$, we have $\operatorname{deg} f=k+1$, and thus $f$ satisfies (i) (with $k+1$ in place of $k$ ). 
We note that the bound $k+1$ in (ii) cannot be replaced by $k$, as the following example shows. Let $F_{\omega}$ denote the free Abelian group generated by countable infinitely many generators. We shall represent $F_{\omega}$ as

$$
F_{\omega}=\left\{\left(t_{1}, t_{2}, \ldots\right): t_{i} \in \mathbb{Z}(i=1,2, \ldots), \exists i_{0}, t_{i}=0\left(i>i_{0}\right)\right\},
$$

where the sum of the elements $\left(t_{1}, t_{2}, \ldots\right)$ and $\left(y_{1}, y_{2}, \ldots\right)$ is $\left(t_{1}+y_{1}, t_{2}+y_{2}, \ldots\right)$. We take the discrete topology on $F_{\omega}$.

Let $q(x)=\sum_{i=1}^{\infty} t_{i}^{2}$ for every $x=\left(t_{1}, t_{2}, \ldots\right) \in F_{\omega}$. Then $q \in \mathrm{GP}$ and $\operatorname{deg} q=2$. We prove that $q\left(x_{1}+\ldots+x_{n}\right)$ is not decomposable of order 2 for any $n \geq 2$. Indeed, $q\left(x_{1}+x_{2}\right)$ is not decomposable (of any order), since otherwise the translates of $q$ would span a linear space of finite dimension, which is not the case (see [7]). Let $n \geq 3$, and suppose that $q\left(x_{1}+\ldots+x_{n}\right)$ is decomposable of order 2 . Then we have

$$
q\left(x_{1}+\ldots+x_{n}\right)=u_{1} \cdot v_{1}+u_{2} \cdot v_{2},
$$

where the function $u_{i}$ only depends on the variables belonging to a set $\emptyset \neq E_{i} \subsetneq\left\{x_{1}, \ldots, x_{n}\right\}$, and $v_{i}$ only depends on the variables belonging to $\left\{x_{1}, \ldots, x_{n}\right\} \backslash E_{i}(i=1,2)$. It is easy to check that there are indices $1 \leq j<k \leq n$ such that $x_{j}$ and $x_{k}$ are separated by both $E_{1}$ and $E_{2}$; that is, $x_{j} \in E_{1}$ and $x_{k} \notin E_{1}$ or the other way around, and $x_{j} \in E_{2}$ and $x_{k} \notin E_{2}$ or the other way around. Fixing $x_{i}$ at zero for every $i \neq j, k$ we can see that $q\left(x_{j}+x_{k}\right)$ is decomposable of order 2 , which is impossible.

The following result gives the direction (i) $\Longrightarrow$ (ii) of Theorem 1 ,

Theorem 3. If $f \in C(G)$ is such that $f\left(x_{1}+\ldots+x_{n}\right)$ is decomposable for some $n \geq 2$, then $f \in \mathrm{GEP}$. More precisely, if $f\left(x_{1}+\ldots+x_{n}\right)$ is decomposable of order $k$, then $f \in \mathrm{GEP}$, and $\operatorname{deg} f \leq k$.

As (i) of Theorem 2 shows, the upper bound $\operatorname{deg} f \leq k$ is sharp in Theorem 3. We prove Theorem 3 in Section 3, using the results of the next section. 


\section{Lemmas}

In this section we assume that $G$ is a commutative unital semigroup written additively. A function $f: G \rightarrow \mathbb{C}$ is a polynomial, if $f=P\left(a_{1}, \ldots, a_{n}\right)$, where $P \in \mathbb{C}\left[x_{1}, \ldots, x_{n}\right]$ and $a_{1}, \ldots, a_{n}$ are continuous additive functions mapping $G$ into $\mathbb{C}$. (These functions are called normal polynomials in [6].) The functions of the form $\sum_{i=1}^{k} p_{i} \cdot m_{i}$, where $p_{i}$ is a polynomial and $m_{i}$ is an exponential for every $i=1, \ldots, k$ are called exponential polynomials. If $f \in C(G)$ then we denote by $V_{f}$ the linear span of the translates of $f$.

Proposition 4. If $f$ is an exponential polynomial, then $\operatorname{deg} f \leq \operatorname{dim} V_{f}$.

Proof. If $f$ is a polynomial, then even the stronger statement $\operatorname{deg} f<\operatorname{dim} V_{f}$ follows from [6, Lemma 2.8, p. 30]. We give a simple direct proof. We prove by induction on $n=\operatorname{deg} f$. If $n=-1$, then $f=0, V_{f}=\{0\}$, and $\operatorname{deg} f=$ $-1<0=\operatorname{dim} V_{f}$. Let $n=\operatorname{deg} f \geq 0$, and suppose that the statement is true for smaller degrees. There exists an $h \in G$ such that $\operatorname{deg} \Delta_{h} f=n-1$. Fixing such an $h$ we find that $f \notin V_{\Delta_{h} f}$, since the elements of $V_{\Delta_{h} f}$ are of degree $\leq n-1$. As $V_{\Delta_{h} f}+\langle f\rangle \subset V_{f}$, it follows that $\operatorname{dim} V_{f}>\operatorname{dim} V_{\Delta_{h} f}$. By the induction hypothesis we have $\operatorname{dim} V_{\Delta_{h} f}>\operatorname{deg} \Delta_{h} f=n-1$, and thus $\operatorname{dim} V_{f}>n$. This proves the statement for polynomials.

To prove the general statement, let $f=\sum_{i=1}^{n} p_{i} \cdot m_{i}$, where $m_{1}, \ldots, m_{n}$ are distinct exponentials and $p_{1}, \ldots, p_{n}$ are nonzero polynomials. For every $i$, we have $V_{p_{i} \cdot m_{i}}=m_{i} \cdot V_{p_{i}}=\left\{m_{i} \cdot p: p \in V_{p_{i}}\right\}$, and thus

$$
\operatorname{dim} V_{p_{i} \cdot m_{i}}=\operatorname{dim} V_{p_{i}} \geq 1+\operatorname{deg} p_{i} .
$$

It is well-known that $p_{i} \cdot m_{i} \in V_{f}$ for every $i$. (See, e.g., [4, Lemma 6]. Although the lemma is stated for groups, the proof works in every Abelian semigroup.) Then we have $V_{f}=V_{p_{1} \cdot m_{1}}+\ldots+V_{p_{n} \cdot m_{n}}$, where the right hand side is a direct sum, as $V_{p_{i} \cdot m_{i}} \cap \sum_{j \neq i} V_{p_{j} \cdot m_{j}}=\{0\}$ for every $i=1, \ldots, n$. Therefore, we obtain

$$
\operatorname{dim} V_{f}=\sum_{i=1}^{n} \operatorname{dim} V_{p_{i} \cdot m_{i}} \geq \sum_{i=1}^{n}\left(1+\operatorname{deg} p_{i}\right) \geq \operatorname{deg} f .
$$


We shall need the notion of modified difference operators introduced by Almira and Székelyhidi in [2]. If $f, \phi: G \rightarrow \mathbb{C}$ and $h \in G$, then we put $\Delta_{\phi, h} f(x)=f(x+h)-\phi(h) \cdot f(x)$ for every $x \in G$. The statement of the following lemma is a consequence of [2, Theorem 17] and of [6, Theorem 8.12, p. 68]. In order to make these note as self-contained as possible, we give an independent direct proof.

Lemma 5. (i) Let $f=\sum_{i=1}^{k} p_{i} \cdot m_{i}$, where $p_{1}, \ldots, p_{k}$ are generalized polynomials and $m_{1}, \ldots, m_{k}$ are exponentials. If $n_{i}>\operatorname{deg} p_{i}$ for every $i=1, \ldots, k$, then we have

$$
\Delta_{m_{1}, h_{1}}^{n_{1}} \ldots \Delta_{m_{k}, h_{k}}^{n_{k}} f=0
$$

for every $h_{1}, \ldots, h_{k} \in G$.

(ii) Let $m_{1}, \ldots, m_{k}$ be different exponentials, and let $n_{1}, \ldots, n_{k}$ be nonnegative integers. If $f: G \rightarrow \mathbb{C}$ is such that (2) holds for every $h_{1}, \ldots, h_{k} \in$ $G$, then $f=\sum_{i=1}^{k} p_{i} \cdot m_{i}$, where $p_{1}, \ldots, p_{k}$ are generalized polynomials, and $\operatorname{deg} p_{i}<n_{i}$ for every $i=1, \ldots, k$.

Proof. (i) If $m$ is an exponential, then we have

$$
\begin{aligned}
\Delta_{m, h} p_{i} \cdot m_{i} & =p_{i}(x+h) \cdot m_{i}(x) \cdot m_{i}(h)-m(h) \cdot p_{i}(x) \cdot m_{i}(x)= \\
& =\left[p_{i}(x+h) \cdot m_{i}(h)-p_{i}(x) \cdot m(h)\right] \cdot m_{i}(x)= \\
& =q(x) \cdot m_{i}(x),
\end{aligned}
$$

where $q \in \mathrm{GP}, \operatorname{deg} q \leq \operatorname{deg} p_{i}$, and $\operatorname{deg} q<\operatorname{deg} p_{i}$ if $m=m_{i}$. From this observation the statement follows by induction on $\sum_{i=1}^{k} \operatorname{deg} p_{i}$.

(ii) First we prove the statement for $k=1$. We have to prove that if $m$ is an exponential and $\Delta_{m, h}^{n} f=0$ for every $h \in G$, then $f=p \cdot m$, where $p \in \mathrm{GP}$ and $\operatorname{deg} p<n$. If $n=0$, then we interpret the condition as $f=0$. In this case we have $f=0 \cdot m$, where $\operatorname{deg} 0=-1$, and so the statement is true. Let $n>0$. It is easy to see that for every $f: G \rightarrow \mathbb{C}$ we have $\Delta_{m, h} f(x)=m(h) \cdot m(x) \cdot \Delta_{h}(f / m)(x)$. Therefore, by induction we find

$$
\Delta_{m, h}^{n} f=m(h)^{n} \cdot m \cdot \Delta_{h}^{n}(f / m) \quad(h \in G) .
$$

If $\Delta_{m, h}^{n} f=0$ for every $h$, then we obtain $\Delta_{h}^{n}(f / m)=0$ for every $h$. By Djoković's theorem this condition implies $f / m \in \mathrm{GP}$ and $\operatorname{deg} p \leq n-1$ (see 
[3, Corollary 1]). Then, by $f=(f / m) \cdot m$, we obtain the statement of (ii) for $k=1$.

Suppose that $k>1$ and the statement is true for $k-1$. We argue by induction on $n=n_{1}+\ldots+n_{k}$. If $n=0$, then $f=0=\sum_{i=1}^{k} 0 \cdot m_{i}$, and the statement is true. Suppose that $n>0$ and that the statement is true for $n-1$. By symmetry, we may assume $n_{1}>0$. Let $f$ be a function satisfying the condition of (ii). Let $h_{2}, \ldots, h_{k} \in G$ be fixed, and put

$$
f_{1}=\Delta_{m_{2}, h_{2}}^{n_{2}} \ldots \Delta_{m_{k}, h_{k}}^{n_{k}} f
$$

Then $\Delta_{m_{1}, h_{1}}^{n_{1}} f_{1}=0$ for every $h_{1} \in G$. As we saw above, this implies $f_{1}=$ $p \cdot m_{1}$, where $p \in \mathrm{GP}$ and $\operatorname{deg} p<n_{1}$. Then $\Delta_{h} \Delta_{h_{1}}^{n_{1}-1} p=0$ for every $h, h_{1} \in G$, and thus $\Delta_{m_{1}, h} \Delta_{m_{1}, h_{1}}^{n_{1}-1} f_{1}=0$ for every $h, h_{1} \in G$ by (3) .

Fix a $h$ such that $m_{1}(h) \neq m_{2}(h)$. Since the difference operators commute, we find that

$$
\Delta_{m_{1}, h_{1}}^{n_{1}-1} \Delta_{m_{2}, h_{2}}^{n_{2}} \ldots \Delta_{m_{k}, h_{k}}^{n_{k}} \Delta_{m_{1}, h} f=0
$$

for every $h_{1}, \ldots, h_{k}$. By the induction hypothesis, this implies

$$
\Delta_{m_{1}, h} f=\sum_{i=1}^{k} p_{i} \cdot m_{i}
$$

where $p_{i} \in \mathrm{GP}$ and $\operatorname{deg} p_{i}<n_{i}$ for every $i$. By the same argument we find that

$$
\Delta_{m_{2}, h} f=\sum_{i=1}^{k} q_{i} \cdot m_{i}
$$

where $q_{i} \in \mathrm{GP}$ and $\operatorname{deg} q_{i}<n_{i}$ for every $i$. We have

$$
\Delta_{m_{1}, h} f(x)-\Delta_{m_{2}, h} f(x)=\left(m_{2}(h)-m_{1}(h)\right) \cdot f(x),
$$

and thus, subtracting (5) from (41) we get

$$
\left(m_{2}(h)-m_{1}(h)\right) \cdot f=\sum_{i=1}^{k}\left(p_{i}-q_{i}\right) \cdot m_{i} .
$$

Dividing by $\left(m_{2}(h)-m_{1}(h)\right)$ we obtain the statement of (ii). 
Lemma 6. Let $V$ be a linear subspace of GEP such that $V \neq\{0\}$, and $\operatorname{deg} f \leq N<\infty$ for every $f \in V$. Then there are exponentials $m_{1}, \ldots, m_{k}$ and positive integers $n_{1}, \ldots, n_{k}$ such that

(i) $\sum_{i=1}^{k} n_{i} \leq \begin{cases}N & \text { if } 1 \notin\left\{m_{1}, \ldots, m_{k}\right\} \text {, } \\ N+1 & \text { if } 1 \in\left\{m_{1}, \ldots, m_{k}\right\} \text {; }\end{cases}$

(ii) every $f \in V$ is of the form $\sum_{i=1}^{k} q_{i} \cdot m_{i}$, where $q_{i} \in \mathrm{GP}$ and $\operatorname{deg} q_{i}<n_{i}$ $(i=1, \ldots, k)$;

(iii) there exists a function $f_{0} \in V$ such that $f_{0}=\sum_{i=1}^{k} p_{i} \cdot m_{i}$, where $p_{i} \in \mathrm{GP}$ and $\operatorname{deg} p_{i}=n_{i}-1$ for every $i=1, \ldots, k$, and

(iv) (2) holds for every $f \in V$ and for every $h_{1}, \ldots, h_{k} \in G$.

Proof. If $f=\sum_{i=1}^{s} p_{i} \cdot m_{i}$, where $m_{1}, \ldots, m_{s}$ are distinct exponentials and $p_{1}, \ldots, p_{s}$ are nonzero generalized polynomials, then we define the spectrum of $f$ as $\operatorname{sp} f=\left\{m_{1}, \ldots, m_{s}\right\}$. If $f=0$, then we let $\operatorname{sp} f=\emptyset$.

Put $S=\bigcup_{f \in V} \operatorname{sp} f$. We show that $S$ has at most $N+1$ elements. Suppose this is not true. Then there are functions $f_{1}, \ldots, f_{n} \in V$ such that

$$
\bigcup_{i=1}^{n} \operatorname{sp} f_{i}=\left\{m_{1}, \ldots, m_{s}\right\}
$$

where $m_{1}, \ldots, m_{s}$ are distinct exponentials, and $s \geq N+2$. Let $f_{i}=$ $\sum_{j=1}^{s} p_{i, j} \cdot m_{j}(i=1, \ldots, n)$, where $p_{i, j} \in \mathrm{GP}$ for every $i=1, \ldots, n$ and $j=1, \ldots, s$. It follows from (66) that for every $j=1, \ldots, s$ there is an $i$ such that $p_{i, j} \neq 0$. If $c_{1}, \ldots, c_{n} \in \mathbb{C}$, then

$$
\sum_{i=1}^{n} c_{i} \cdot f_{i}=\sum_{j=1}^{s}\left(\sum_{i=1}^{n} c_{i} \cdot p_{i, j}\right) \cdot m_{j}
$$

belongs to $V$. For every $j$, the set of $n$-tuples $\left(c_{1}, \ldots, c_{n}\right)$ with $\sum_{i=1}^{n} c_{i} \cdot p_{i, j}=0$ constitutes a linear subspace $L_{j}$ of $\mathbb{C}^{n}$. The linear subspace $L_{j}$ is proper, since $p_{i, j} \neq 0$ for a suitable $i$, and thus $\left(\delta_{1, i}, \ldots, \delta_{n, i}\right) \notin L_{j}$, where $\delta_{k, i}$ is the Kronecker delta. Since $\bigcup_{j=1}^{s} L_{j} \subsetneq \mathbb{C}^{n}$, there is an $n$-tuple $\left(c_{1}, \ldots, c_{n}\right)$ such that $\sum_{i=1}^{n} c_{i} \cdot p_{i, j} \neq 0$ for every $j=1, \ldots, s$, and then the spectrum 
of $f=\sum_{i=1}^{n} c_{i} \cdot f_{i}$ equals $\left\{m_{1}, \ldots, m_{s}\right\}$. Then $\operatorname{deg} f \geq s-1>N$, which contradicts the condition on $V$.

We proved that $S$ has at most $N+1$ elements. Let $S=\left\{m_{1}, \ldots, m_{k}\right\}$, where $m_{1}, \ldots, m_{k}$ are distinct exponentials, and $k \leq N+1$. Then every $f \in V$ is of the form $\sum_{i=1}^{k} q_{i} \cdot m_{i}$, where $q_{i}=q_{i}(f) \in$ GP for every $i=1, \ldots, k$. Since $\operatorname{deg} f \leq N$ for every $f \in V$, it follows that $\operatorname{deg} q_{i}(f) \leq N$ for every $f \in V$ and for every $i=1, \ldots, k$. Let $n_{i}=1+\max \left\{\operatorname{deg} q_{i}(f): f \in V\right\}$ for every $i=1, \ldots, k$, and let $f_{i} \in V$ be such that $\operatorname{deg} q_{i}\left(f_{i}\right)=n_{i}-1(i=1, \ldots, k)$.

The set of $k$-tuples $\left(c_{1}, \ldots, c_{k}\right) \in \mathbb{C}^{k}$ such that $\operatorname{deg}\left(\sum_{j=1}^{k} c_{j} \cdot q_{i}\left(f_{j}\right)\right)<$ $n_{i}-1$ is a linear subspace $M_{i}$ of $\mathbb{C}^{k}$ for every $i=1, \ldots, k$. We have $M_{i} \subsetneq \mathbb{C}^{k}$, as $\left(\delta_{1, i}, \ldots, \delta_{n, i}\right) \notin M_{i}$. Thus $M_{i}$ is a proper subspace of $\mathbb{C}^{k}$ for every $i$. Therefore, we have $\bigcup_{i=1}^{k} M_{i} \subsetneq \mathbb{C}^{k}$, and there is an $k$-tuple $\left(c_{1}, \ldots, c_{k}\right)$ such that $\operatorname{deg}\left(\sum_{j=1}^{k} c_{j} \cdot q_{i}\left(f_{j}\right)\right)=n_{i}-1$ for every $i=1, \ldots, k$. Put $f_{0}=\sum_{j=1}^{k} c_{j}$. $f_{j}$; then $f_{0} \in V$, and (iii) holds.

We have $f_{0}=\sum_{i=1}^{k} p_{i} \cdot m_{i}$, where $p_{1}, \ldots, p_{k}$ are nonzero generalized polynomials with $\operatorname{deg} p_{i}=n_{i}-1(i=1, \ldots, k)$. If $1 \notin\left\{m_{1}, \ldots, m_{k}\right\}$, then $N \geq \operatorname{deg} f_{0}=\sum_{i=1}^{k} n_{i}$. On the other hand, if $1 \in\left\{m_{1}, \ldots, m_{k}\right\}$, then $N \geq \operatorname{deg} f_{0}=-1+\sum_{i=1}^{k} n_{i}$, proving (i). Statement (ii) is obvious from the construction. Finally, (iv) follows from (ii) and from Lemma 5 .

Lemma 7. Let $n, N$ be positive integers, and suppose that

(i) $V$ is a linear subspace of GEP such that $\operatorname{deg} f \leq N$ for every $f \in V$, and

(ii) $W$ is a linear subspace of $C(G)$ such that $\operatorname{dim} W \leq n$.

Then the number of exponentials contained in $W+V$ is at most $n+N+1$.

Proof. By the previous lemma, there are exponentials $m_{1}, \ldots, m_{k}$ such that $k \leq N+1$, and every $f \in V$ is of the form $\sum_{i=1}^{k} q_{i} \cdot m_{i}$, where $q_{1}, \ldots, q_{k} \in$ GP. We prove that there are at most $n$ exponentials in $W+V$ different from $m_{1}, \ldots, m_{k}$. 
Suppose this is not true, and let $e_{1}, \ldots, e_{n+1} \in W+V$ be distinct exponentials different from $m_{1}, \ldots, m_{k}$. Let $e_{i}=w_{i}+v_{i}$, where $w_{i} \in W$ and $v_{i} \in V$ for every $i=1, \ldots, n+1$. Since $\operatorname{dim} W \leq n$, the elements $w_{1}, \ldots, w_{n+1}$ are linearly dependent. That is, there are complex numbers $c_{1}, \ldots, c_{n+1}$, not all zero, such that $\sum_{j=1}^{n+1} c_{j} w_{j}=0$. Then

$$
\sum_{j=1}^{n+1} c_{j} e_{j}=\sum_{j=1}^{n+1} c_{j} v_{j} \in V .
$$

Therefore, we have $\sum_{j=1}^{n+1} c_{j} e_{j}=\sum_{i=1}^{k} q_{i} \cdot m_{i}$ with suitable $q_{1}, \ldots, q_{k} \in \mathrm{GP}$. However, the representation of this form is unique, and thus, if $c_{j} \neq 0$ for a $j$, then $e_{j}=m_{i}$ for some $i=1, \ldots, k$, which is impossible.

Lemma 8. Let $n, N$ be positive integers, and suppose that

(i) $V$ is a linear subspace of GEP such that $\operatorname{deg} f \leq N$ for every $f \in V$,

(ii) $W$ is a linear subspace of $C(G)$ such that $\operatorname{dim} W \leq n$, and

(iii) $F$ is a translation invariant linear subspace of $W+V$.

Then $F \subset$ GEP, and $\operatorname{deg} f \leq n+N$ for every $f \in F$.

Proof. Let $m_{1}, \ldots, m_{k}$ and $n_{1}, \ldots, n_{k}$ be as in Lemma 6. Then we have $\sum_{i=1}^{k} n_{i} \leq N+1$ or $\sum_{i=1}^{k} n_{i} \leq N$ according to whether or not $m_{i}=1$ for one of the indices $i=1, \ldots, k$. By Lemma 7 , the number of exponentials contained in $W+V$ is finite. Let $m_{k+1}, \ldots, m_{k+s}$ be the exponentials contained in $W+V$ and different from $m_{1}, \ldots, m_{k}$.

We show that every function in $F \cap$ GEP is of the form $\sum_{i=1}^{k+s} q_{i} \cdot m_{i}$, where $q_{i} \in$ GP for every $i$. Suppose $f \in F \cap$ GEP and $f=\sum_{j=1}^{i=1} p_{j} \cdot e_{j}$, where $e_{1}, \ldots, e_{t}$ are distinct exponentials and $p_{1}, \ldots, p_{t} \in \mathrm{GP} \backslash\{0\}$. Since $F$ is a translation invariant linear space, it follows that $e_{j} \in F \subset W+V$ for every $j$. Thus $e_{j}$ equals one of $m_{1}, \ldots, m_{k+s}$ for every $j$, proving the statement.

For every $h_{1}, \ldots, h_{k} \in G$ we put $h=\left(h_{1}, \ldots, h_{k}\right)$ and

$$
\Delta_{(h)}=\Delta_{m_{1}, h_{1}}^{n_{1}} \ldots \Delta_{m_{k}, h_{k}}^{n_{k}} .
$$


Fix $h_{1}, \ldots, h_{k}$. By the choice of the numbers $n_{i}$ and by (iv) of Lemma 6, we have $\Delta_{(h)} v=0$ for every $v \in V$.

It is clear that $F_{(h)}=\left\{\Delta_{(h)} f: f \in F\right\}$ is a translation invariant linear subspace of $F$. If $f \in F$, then $f=w+v$ for some $w \in W$ and $v \in V$. Then $\Delta_{(h)} f=\Delta_{(h)} w+\Delta_{(h)} v=\Delta_{(h)} w$, and thus $F_{(h)}$ is a linear subspace of $W_{(h)}=\left\{\Delta_{(h)} w: w \in W\right\}$. Clearly, $W_{(h)}$ is of dimension $\leq n$, and then the same is true for $F_{(h)}$.

Therefore, by a well-known theorem (see [6, Theorem 10.1] and the references given on [6, p. 79]), each element of $F_{(h)}$ is an exponential polynomial. By Proposition 4, $\operatorname{deg} \phi \leq n$ for every $\phi \in F_{(h)}$. Therefore, by (i) of Lemma 5. we have

$$
\Delta_{m_{1}, h_{1}}^{n+1} \ldots \Delta_{m_{k+s}, h_{k+s}}^{n+1} \Delta_{(h)} \phi=0
$$

for every $\phi \in F_{(h)}$ and for every $h_{k+1}, \ldots, h_{k+s} \in G$. According to the definition of $\Delta_{(h)}$, this implies

$$
\Delta_{m_{1}, h_{1}}^{n_{1}+n+1} \ldots \Delta_{m_{k}, h_{k}}^{n_{k}+n+1} \Delta_{m_{k+1}, h_{k+1}}^{n+1} \ldots \Delta_{m_{k+s}, h_{k+s}}^{n+1} f=0
$$

for every $f \in F$ and for every $h_{1}, \ldots, h_{k+s} \in G$. Then, by (ii) of Lemma 5, it follows that $F \subset$ GEP and $\sup _{f \in F} \operatorname{deg} f<\infty$. We show that $\operatorname{deg} f \leq n+N$ for every $f \in F$.

Applying Lemma [6] with $F$ in place of $V$, we find a set of indices $J \subset$ $\{1, \ldots, k+s\}$ and positive numbers $d_{i}(i \in J)$ such that every $f \in F$ is of the form $\sum_{i \in J} q_{i} \cdot m_{i}$, where $q_{i} \in \mathrm{GP}$ and $\operatorname{deg} q_{i}<d_{i}(i \in J)$, and there is a function $f_{0} \in F$ such that $f_{0}=\sum_{i \in J} p_{i} \cdot m_{i}$, where $p_{i} \in$ GP and $\operatorname{deg} p_{i}=d_{i}-1(i \in J)$. Therefore, we have $\sum_{i \in J} d_{i}=\operatorname{deg} f_{0}+1$ or $\sum_{i=1}^{k+s} d_{i}=\operatorname{deg} f_{0}$ according to whether or not $m_{i}=1$ for one of the indices $i \in J$.

We put $n_{i}=0$ for every $i \in J \backslash\{1, \ldots, k\}$. We prove that

$$
\sum_{i \in J}\left(d_{i}-n_{i}\right) \leq n
$$

Suppose this is not true, and let $I$ denote the set of indices $i \in J$ such that $d_{i}>n_{i}$. Then

$$
\sum_{i \in I}\left(d_{i}-n_{i}\right) \geq \sum_{i \in J}\left(d_{i}-n_{i}\right)>n .
$$


Since $f_{0}=\sum_{i \in J} p_{i} \cdot m_{i} \in F$ and $f$ is a translation invariant linear space, we have $p_{i} \cdot m_{i} \in F$ for every $i \in J$. Moreover, $V_{p_{i} \cdot m_{i}}=m_{i} \cdot V_{p_{i}} \subset F$ for every $i \in J$. For every $p \in \mathrm{GP}$ we have $\operatorname{deg} \Delta_{h} p=(\operatorname{deg} p)-1$ for a suitable $h \in G$, and thus $V_{p}$ contains generalized polynomials of any degree between 0 and $\operatorname{deg} p$. Let $p_{i, j} \in V_{p_{i}}$ be such that $\operatorname{deg} p_{i, j}=j$ for every $i \in I$ and $n_{i} \leq j \leq d_{i}-1$.

Let $f_{1}, \ldots, f_{t}$ be an enumeration of the functions $p_{i, j} \cdot m_{i}$ with $i \in I$ and $n_{i} \leq j \leq d_{i}-1$. Then the functions $f_{1}, \ldots, f_{t}$ belong to $F$, and $t=$ $\sum_{i \in I}\left(d_{i}-n_{i}\right)>n$ by (8) . Since $f_{1}, \ldots, f_{t} \in F$, we have $f_{\nu}=w_{\nu}+v_{\nu}$, where $w_{\nu} \in W, v_{\nu} \in V(\nu=1, \ldots, t)$. Now $\operatorname{dim} W \leq n<t$ implies that there are complex numbers $c_{1}, \ldots, c_{t}$, not all zero, such that $\sum_{\nu=1}^{t} c_{\nu} \cdot w_{\nu}=0$. Let $f=\sum_{\nu=1}^{t} c_{\nu} \cdot f_{\nu}$. Then $f \in F$, and $f=\sum_{\nu=1}^{t} c_{\nu} \cdot v_{\nu} \in V$. On the other hand,

$$
f=\sum_{\nu=1}^{t} c_{\nu} \cdot f_{\nu}=\sum_{i \in I} \sum_{j=n_{i}}^{d_{i}-1} c_{i, j} \cdot p_{i, j} \cdot m_{i},
$$

where $c_{i, j}\left(i \in I, n_{i} \leq j \leq d_{i}-1\right)$ is an enumeration of the numbers $c_{\nu}$ $(\nu=1, \ldots, t)$. Thus $f=\sum_{i \in I} q_{i} \cdot m_{i}$, where $q_{i}=\sum_{j=n_{i}}^{d_{i}-1} c_{i, j} \cdot p_{i, j}(i \in I)$. If $i \in I$ is such that $c_{i, j}$ is nonzero for at least one $n_{i} \leq j \leq d_{i}-1$, then $\operatorname{deg} q_{i} \geq n_{i}$. If $1 \leq i \leq k$, then $\operatorname{deg} q_{i}<n_{i}$ by the choice of $n_{i}$, which is impossible. If $i \in J \backslash\{1, \ldots, k\}$, then $n_{i}=0$, and thus $\operatorname{deg} q_{i} \geq n_{i}$ implies $q_{i} \neq 0$, contradicting the choice of $m_{1}, \ldots, m_{k}$. Both cases are impossible, so we must have (7).

Now we consider three cases. First we assume that $m_{i} \neq 1$ for every $i \in J \cup\{1, \ldots, k\}$. Then we have $\sum_{i=1}^{k} n_{i} \leq N$ and thus, by (7), we obtain

$$
\operatorname{deg} f_{0}=\sum_{i \in J} d_{i} \leq n+\sum_{i \in J} n_{i} \leq n+\sum_{i=1}^{k} n_{i} \leq n+N .
$$

Next consider the case when $m_{i}=1$ for some $i \in J$. Then we have $\sum_{i=1}^{k} n_{i} \leq$ $N+1$ and thus, by (7), we obtain

$$
\operatorname{deg} f_{0}=-1+\sum_{i \in J} d_{i} \leq n-1+\sum_{i \in J} n_{i} \leq n-1+\sum_{i=1}^{k} n_{i} \leq n+N .
$$


Finally, if $m_{i}=1$ for some $i \in\{1, \ldots, k\} \backslash J$, Then we have

$$
\sum_{i \in J} n_{i}<\sum_{i=1}^{k} n_{i} \leq N+1
$$

and thus, by (17), we obtain

$$
\operatorname{deg} f_{0}=\sum_{i \in J} d_{i} \leq n+\sum_{i \in J} n_{i} \leq n+N
$$

Summing up: we have $\operatorname{deg} f_{0} \leq n+N$ in each of the cases. Therefore, we have $\operatorname{deg} f \leq \operatorname{deg} f_{0} \leq n+N$ for every $f \in F$.

\section{Proof of Theorem 3}

We follow the argument of the proof of [5, Theorem 6]. We prove by induction on $n$. If $n=2$ and $f\left(x_{1}+x_{2}\right)$ is decomposable of order $k$, then $f\left(x_{1}+x_{2}\right)=$ $\sum_{i=1}^{k} u_{i}\left(x_{1}\right) \cdot v_{i}\left(x_{2}\right)$ for every $x_{1}, x_{2} \in G$. This implies that the translates of $f$ belong to the linear space generated by the functions $u_{1}, \ldots, u_{k}$, and thus $V_{f}$ is of dimension $\leq k$. Therefore, $f$ is an exponential polynomial, and $\operatorname{deg} f \leq k$ by Proposition 4 .

Let $n>2$, and suppose that the statement is true for $n-1$. Suppose that $f\left(x_{1}+\ldots+x_{n}\right)=\sum_{i=1}^{k} u_{i} \cdot v_{i}$, where $u_{i}$ only depends on the variables belonging to a nonempty set $E_{i} \subsetneq\left\{x_{1}, \ldots, x_{n}\right\}$, and $v_{i}$ only depends on the variables belonging to $\left\{x_{1}, \ldots, x_{n}\right\} \backslash E_{i}(i=1, \ldots, k)$. Switching $u_{i}$ and $v_{i}$ if necessary, we may assume that $x_{n} \notin E_{i}$ for every $i$. We may also assume that $E_{i}=\left\{x_{1}, \ldots, x_{n-1}\right\}$ for every $i \leq p$, and $E_{i} \subsetneq\left\{x_{1}, \ldots, x_{n-1}\right\}$ for $p<i \leq k$.

If $p=0$, then substituting $x_{n}=0$ in $f\left(x_{1}+\ldots+x_{n}\right)=\sum_{i=1}^{k} u_{i} \cdot v_{i}$ we can see that $f\left(x_{1}+\ldots+x_{n-1}\right)$ is decomposable of order $k$. Then, by the induction hypothesis, we have $f \in \mathrm{GEP}$, and $\operatorname{deg} f \leq k$, and we are done. Therefore, we may assume that $p>0$. Note that the functions $v_{1}, \ldots, v_{p}$ only depend on $x_{n}$. We have

$$
\sum_{i=1}^{p} u_{i} \cdot v_{i}=f\left(x_{1}+\ldots+x_{n}\right)-\sum_{j=p+1}^{k} u_{j} \cdot v_{j} .
$$


We may assume that the functions $v_{1}, \ldots, v_{p}$ are linearly independent. Then there are elements $g_{1}, \ldots, g_{p} \in G$ such that the determinant $\operatorname{det}\left|v_{i}\left(g_{j}\right)\right|$ is nonzero (see [1, Lemma 1, p. 229]). Substituting $x_{n}=g_{\nu}$ into (9) for every $\nu=1, \ldots, p$ we obtain a system of linear equations with unknowns $u_{1}, \ldots, u_{p}$. Since the determinant of the system is nonzero, we find that each of $u_{1}, \ldots, u_{p}$ is a linear combination of the right hand sides. None of $u_{1}, \ldots, u_{k}$ depends on $x_{n}$, so we obtain

$$
u_{i}=f_{i}\left(x_{1}+\ldots+x_{n-1}\right)+\sum_{j=p+1}^{k} u_{j} \cdot w_{i, j} \quad(i=1, \ldots, p),
$$

where $f_{1}, \ldots, f_{p}$ are functions of one variable mapping $G$ into $\mathbb{C}$, and $w_{i, j}$ only depends on the variables belonging to the nonempty set $\left\{x_{1}, \ldots, x_{n-1}\right\} \backslash E_{j}$ for every $i=1, \ldots, p$ and $j=p+1, \ldots, k$.

Substituting the right hand sides of (10) into $f\left(x_{1}+\ldots+x_{n}\right)=\sum_{i=1}^{k} u_{i} \cdot v_{i}$ and rearranging the terms we obtain

$$
\begin{aligned}
& f\left(x_{1}+\ldots+x_{n}\right)-\sum_{i=1}^{p} f_{i}\left(x_{1}+\ldots+x_{n-1}\right) \cdot v_{i}\left(x_{n}\right)= \\
& =\sum_{i=1}^{p} \sum_{j=p+1}^{k} u_{j} \cdot w_{i, j} \cdot v_{i}\left(x_{n}\right)+\sum_{i=p+1}^{k} u_{j} \cdot v_{j}=\sum_{j=p+1}^{k} u_{j} \cdot z_{j},
\end{aligned}
$$

where $z_{j}=\sum_{i=1}^{p}\left(w_{i, j} \cdot v_{i}\left(x_{n}\right)+v_{j}\right)$. Note that, for every $j=p+1, \ldots, k$, $z_{j}$ only depends on the variables belonging to $\left\{x_{1}, \ldots, x_{n}\right\} \backslash E_{j}$, since each function appearing in the definition of $z_{j}$ has this property.

Let $\Sigma$ denote the set of $(k+1)$-tuples $\left(\phi, a_{1}, \ldots, a_{p}, b_{p+1}, \ldots, b_{k}\right)$ satisfying the following conditions:

(i) $\phi \in C(G)$ and $a_{1}, \ldots, a_{p}: G \rightarrow \mathbb{C}$,

(ii) for every $j=p+1, \ldots, k, b_{j}: G^{n} \rightarrow \mathbb{C}$ is a function only depending on the variables belonging to $\left\{x_{1}, \ldots, x_{n}\right\} \backslash E_{j}$, and

$$
\phi\left(x_{1}+\ldots+x_{n}\right)-\sum_{i=1}^{p} f_{i}\left(x_{1}+\ldots+x_{n-1}\right) \cdot a_{i}\left(x_{n}\right)=\sum_{j=p+1}^{k} u_{j} \cdot b_{j}
$$


for every $x_{1}, \ldots, x_{n} \in G$.

It is clear that $\Sigma$ is a linear space over $\mathbb{C}$ under addition and multiplication by complex numbers coordinate-wise.

Let $F$ denote the set of functions $\phi \in C(G)$ such that

$$
\left(\phi, a_{1}, \ldots, a_{p}, b_{p+1}, \ldots, b_{k}\right) \in \Sigma
$$

for some $a_{1}, \ldots, a_{p}, b_{p+1}, \ldots, b_{k}$. By (11), we have $f \in F$. Since $\Sigma$ is a linear space over $\mathbb{C}$, so is $F$.

We show that $F$ is translation invariant. Let $\phi \in F$ and $h \in G$ be arbitrary. Let $\left(\phi, a_{1}, \ldots, a_{p}, b_{p+1}, \ldots, b_{k}\right) \in \Sigma$. Then (12) holds for every $x_{1}, \ldots, x_{n} \in G$. Replacing $x_{n}$ by $x_{n}+g$ we obtain

$$
T_{g} \phi\left(x_{1}+\ldots+x_{n}\right)-\sum_{i=1}^{p} f_{i}\left(x_{1}+\ldots+x_{n-1}\right) \cdot T_{g} a_{i}\left(x_{n}\right)=\sum_{j=p+1}^{k} u_{j} \cdot \bar{b}_{j},
$$

where $\bar{b}_{j}=T_{(0, \ldots, 0, g)} b_{j}$. It is clear that $\bar{b}_{j}$ only depends on the variables belonging to $\left\{x_{1}, \ldots, x_{n}\right\} \backslash E_{j}$. Therefore, we have

$$
\left(T_{g} \phi, T_{g} a_{1}, \ldots, T_{g} a_{p}, \bar{b}_{p+1}, \ldots, \bar{b}_{k}\right) \in \Sigma,
$$

and $T_{g} \phi \in F$.

Let $V$ denote the set of functions $\phi-\sum_{i=1}^{p} a_{i}(0) \cdot f_{i}$ such that (13) holds for suitable functions $b_{p+1}, \ldots, b_{k}$. It is clear that $V$ is a linear space. If $v \in V$, then (12) gives

$$
v\left(x_{1}+\ldots+x_{n-1}\right)=\sum_{j=p+1}^{k} u_{j} \cdot \tilde{b}_{j},
$$

where $\tilde{b}_{j}$ is obtained from $b_{j}$ by putting $x_{n}=0$. Since $E_{j}$ is a proper subset of $\left\{x_{1}, \ldots, x_{n-1}\right\}$ for every $j=p+1, \ldots, k$, we find that $v\left(x_{1}+\ldots+x_{n-1}\right)$ is decomposable of order $k-p$. By the induction hypothesis it follows that $V \subset \operatorname{GEP}$ and $\operatorname{deg} v \leq k-p$ for every $v \in V$.

Let $W$ denote the linear span of $f_{1}, \ldots, f_{p}$. It is clear that every $\phi \in F$ is of the form $w+v$, where $w \in W$ and $v \in V$. Therefore, by Lemma 8 , 
we have $\phi \in \mathrm{GEP}$ and $\operatorname{deg} \phi \leq p+(k-p)=k$. Since $f \in F$, the proof is complete.

Acknowledgement The author was supported by the Hungarian National Foundation for Scientific Research, Grant No. K124749.

\section{References}

[1] J. Aczél and, J. Dhombres: Functional equations in several variables. Encyclopedia of Mathematics and its Applications, 31. Cambridge University Press, Cambridge, 1989.

[2] J. M. Almira and L. Székelyhidi, Montel-type theorems for exponential polynomials, Demonstr. Math. 49 (2016), no. 2, 197-212.

[3] D. Ž. Djoković, A representation theorem for $\left(X_{1}-1\right)\left(X_{2}-1\right) \ldots\left(X_{n}-\right.$ 1) and its applications, Ann. Polon. Math. 22 (1969), 189-198.

[4] M. Laczkovich, Local spectral synthesis on Abelian groups, Acta Math. Hungar. 142 (2014), 313-329.

[5] E. Shulman, Decomposable functions and representations of topological semigroups, Aequat. Math. 79 (2010), 13-21.

[6] L. Székelyhidi: Convolution Type Functional Equations on Topological Abelian Groups. World Scientific, 1991.

[7] L. Székelyhidi, The failure of spectral synthesis on some types of discrete Abelian groups, J. Math. Anal. and Applications 291 (2004), $757-763$.

\section{Miklós Laczkovich}

Eötvös Loránd University

Budapest, Hungary

laczk@cs.elte.hu 